\title{
Un método para evaluar el enriquecimiento de metales en sedimentos marinos en Costa Rica
}

\author{
Omar G. Lizano ${ }^{1,2,3,4}$, Eric J. Alfaro ${ }^{1,2,3,4}$ \& Alfonso Salazar Matarrita ${ }^{4,5}$ \\ 1. Centro de Investigación en Ciencias del Mar y Limnología (CIMAR), \\ 2. Centro de Investigaciones Geofísicas (CIGEFI), \\ 3. Departamento de Física Atmosférica, Oceánica y Planetaria (DFAOP), \\ 4. Escuela de Física, \\ 5. Centro de Investigación en Ciencias Atómicas Nucleares y Moleculares (CICANUM). \\ Universidad de Costa Rica. 11501-2060 San José, Costa Rica; omar.lizano@ucr.ac.cr; eric.alfaro@ucr.ac.cr; alfonso. \\ salazar@ucr.ac.cr
}

Recibido 22-VII-2011. Corregido 18-XI-2011. Aceptado 15-II-2012.

\begin{abstract}
A method to evaluate metal enrichment in marine sediments. In order to evaluate metal enrichment in sediments, a method is proposed and tested in Bahia Culebra and the Golfo de Nicoya, Costa Rica through the normalization of the elements against aluminum, and by linear regression of the logarithm of the concentrations of different elements respect to aluminum. The distributions of the elements manganese, and strontium of Bahía Culebra did not satisfy the tests of normalization and linear regression, indicating a nonnatural distribution or enrichment of these elements in this region. In the Golfo de Nicoya the elements copper , zinc, rubidium and the strontium did not satisfy the test of normality or the linear regression with respect to aluminum, indicating a possible enrichment of these elements. The majority of the concentrations of the elements in two sample sites, with the exception of chromium, are within the natural ranges in rocks or clays of marine sediments, and within the concentration ranges of other studies done in these same regions. Chromium has average values beyond the natural concentrations, the values of some samples in the Golfo de Nicoya are up to 10 times greater than the concentration value of a typical bay with high contamination of this element. Rev. Biol. Trop. 60 (Suppl. 2): 197-211. Epub 2012 April 01.
\end{abstract}

Key words: metal concentration, Bahía Culebra, Golfo de Nicoya, enrichment, contamination, Costa Rica, Central America.

La meteorización de rocas en la tierra es una de las mayores fuentes de sedimento en la naturaleza. Los ácidos formados en la atmósfera o durante la descomposición de materia orgánica (por ejemplo, ácido carbónico) forman disoluciones que rompen las rocas y se lleva sus constituyentes en disolución o como partículas sólidas con los minerales (Schropp \& Windom1988, Schropp et al. 1990) A causa de su baja solubilidad, los metales permanecen en la disolución en concentraciones muy bajas. Las partículas sólidas son compuestos principalmente de minerales químicamente resistentes, como cuarzo y minerales secundarios de arcillas, que son los productos de otros minerales de aluminosilicatos (Schropp \& Windom 1988). De esta forma, la mayor parte de las especies de metales transportados por los ríos están fuertemente ligados a las fases sólidas de aluminosilicatos. Como resultado de la meteorización existe muy poco fraccionamiento entre los metales y el aluminio.

Las corrientes de los ríos y sus afluentes que desembocan en el mar, son las responsables del transporte permanente de metales hacia las zonas costeras. Simultáneamente, las corrientes marinas a lo interno de las bahías y estuarios, se encargan de depositarlos en los 
sedimentos marinos. Por lo general, según Ryan \& Windom (1988), una vez que estos constituyentes ingresan en las aguas saladas, floculan y se genera una precipitación desde la columna de agua hacia los sedimentos del fondo marino. De esta manera, el sedimento se convierte en la mayor reserva de metales en los ambientes marinos, a saber:estuarios y bahías.

Las concentraciones de metales en sedimentos marinos tienden a variar espacialmente y su magnitud depende del tamaño de grano, de la fuente mineral, y de la composición química de los sedimentos (Schropp \& Windom 1988, Loring 1991, Cooke \& Drury 1998, Shiff \& Weisberg 1999, Weisberg et al. 2000). Las concentraciones metálicas en sedimentos del fondo, o en la interface agua-sedimento o aún en los sedimentos suspendidos, pueden exceder la concentración de metales en la columna de agua en algunos órdenes de magnitud (Horowitz 1991, Lee \& Jones-Lee 1993). En el pasado sin embargo, los estudios de calidad de agua se enfocaron en estudiar los contaminantes en la columna de agua y se ignoró el hecho de que los sedimentos marinos son el sumidero y reservorio de estos elementos (Horowitz 1991, Loring 1991, Carvalho et al. 2002). Muchos estudios en el pasado, fallaron al no reconocer que la remoción de metales del sedimento contaminado del fondo marino es causa de los problemas de calidad de agua (Carvalho et al. 2002).

La interpretación de datos ambientales de metales pesados se hace difícil por el hecho de que concentraciones absolutas de metales en sedimentos costeros son influidas por una variedad de factores, que incluye mineralogía, tamaño de grano, contenido orgánico y el enriquecimiento antropogénico (Schropp \& Windom 1988). El aluminio es el segundo metal más abundante en la corteza terrestre después del silicio (Schropp \& Windom 1988). Los resultados de diferentes estudios han indicado que las proporciones relativas de metales y aluminio en la corteza terrestre son aproximadamente constantes (Schropp \& Windom1988, Carvalho et al. 2002). Esto no es una sorpresa, pues como se dijo anteriormente, hay poco fraccionamiento de los metales durante los procesos de meteorización. Las técnicas de normalización son las más usadas para interpretar las concentraciones de metales observadas en los sedimentos. Algunas de ellas han incluido el tamaño de grano (Ackerman et al. 1983), el carbón orgánico total (Windom et al. 1989), y los elementos como hierro (Trefry et al. 1985, Daskalakis y O'Connor 1995), aluminio (Hanson et al. 1993, Schropp et al. 1990) y litio (Loring 1990).

La normalización respecto al aluminio ha sido estudiada con mucho éxito (Goldberg et al. 1979, Trefrey et al. 1985, Schropp \& Windom,1988 ), fundamentada en las siguientes razones: es refractario en material sedimentario, la actividad humana influye muy poco, muestra una adecuada correlación con el tamaño de partícula, es el segundo metal más abundante en la corteza terrestre, muestra una relación constante con otros elementos proporcionalmente menos abundantes que se encuentran en el material mineral y en los sedimentos. La normalización de la razón del logaritmo de las concentraciones del elemento/aluminio y la regresión lineal con sus límites de predicción, es una herramienta interpretativa utilizada por varios autores para determinar el enriquecimiento por metales pesados en los sedimentos marinos (Schropp \& Windom 1988, Schropp et al. 1990). Esta metodología toma en cuenta la variabilidad natural de los metales y determina el enriquecimiento respecto a las concentraciones naturales esperadas. Esta herramienta interpretativa se puede utilizar en aplicaciones quer incluye: 1) distinción de las concentraciones naturales y enriquecidas de metales en sedimentos costeros, 2) comparación de las concentraciones de los metales dentro de un sistema estuarino, 3) comparación de las concentraciones de los metales en sistemas estuarinos diferentes, 4) identificación de las fuentes de contaminación, 5) vigilancia de las tendencias en las concentraciones de metales con el tiempo en estos cuerpos de agua, y 6) evaluación del procedimiento de muestreo o errores de laboratorio, como lo señala Schropp \& Windom (1988).

La evaluación de la concentración de metales en sedimentos marinos es una forma 
efectiva de medir el grado de contaminación por metales, ya que los metales en áreas costeras se pueden originar también por actividades humanas. Hoy día, las emisiones antropogénicas pueden ser mayores que las naturales. $\mathrm{Al}$ constituirse los sedimentos en una adecuada trampa para los materiales que se lanzan al ambiente, estos se convierten en un indicador importante para la contaminación ambiental (Ryan \& Windom 1988).

Estudios de metales sobre sedimentos marinos en el Golfo de Nicoya, Costa Rica iniciaron con los trabajos Dean et al. (1986) y Kress et al. (1997). El Golfo de Nicoya es un estuario con la mayor población costera de Costa Rica y recoge las aguas de varias cuencas hidrográficas, algunas de las cuales con gran actividad agrícola, que es una fuente de contaminantes en los estuarios (Mata \& Blanco, 1994). Por otro lado, Bahía Culebra es un cuerpo de agua sin desarrollos urbanos importantes aún, aparentemente no contaminado, por lo que el desarrollo de patrones de contaminación en este cuerpo de agua (Fonseca 2006, GarcíaCéspedes et al. 2004). La comparación entre el Golfo de Nicoya y Bahía Culebra, en cuanto a concentraciones de metales en sedimentos marinos, posee una gran relevancia, ya que la Bahía está siendo transformada rápidamente con un desarrollo hotelero significativo.

Las ecuaciones que se elaboran en la discusión y los resultados gráficos que se presentan en este estudio constituyen la herramienta propuesta para establecer estándares de enriquecimiento/contaminación de los metales: aluminio, potasio, calcio, manganeso, titanio, hierro, cromo, zinc, rubidio, estroncio y plomo en Bahía Culebra y el Golfo de Nicoya. Además, los resultados de nuevos muestreos se podrán comparar con los ya existentes, como la identificación de contaminación de las muestras o errores en los análisis.

\section{MATERIALES Y MÉTODOS}

Se utilizó un bote y una draga Van Veen para extraer 50 muestras de sedimentos marinos superficiales en Bahía Culebra y el Golfo de Nicoya entre marzo 2004 y diciembre 2005 (Fig. 1A, B). Las posiciones de cada estación se determinaron con un GPS manual.

Antes del análisis con el espectrómetro de rayos $\mathrm{X}$, las muestras se sometieron a un proceso de secado, pulverizado en mortero hasta homogenizar. Se separó la fracción menor a $50 \mu \mathrm{m}$. Con un gramo de muestra pulverizada se formó una pastilla del material, tres por muestra, para ser irradiada con rayos $\mathrm{X}$ de un generador Iso-debyeflex 2002 con blanco de Molibdeno a $40 \mathrm{kV}$ y $30 \mathrm{~mA}$.

Se realizó un análisis espectral integral utilizando un sistema de Fluorescencia de Rayos X dispersivo en energía, EDXRF (por sus siglas en inglés Energy Dispersive X Ray Fluorescente). La detección y el conteo de los rayos $\mathrm{X}$ característicos emitidos por los elementos presentes en la muestra y la radiación de retro dispersión, se obtiene con un espectrómetro conformado por el detector $\mathrm{Si}(\mathrm{Li})$, el amplificador y analizador multicanal Nucleus II acoplado a una computadora. El espectro de rayos $\mathrm{X}$ y la valoración de la intensidad de cada fotopico se realiza en forma simultánea con un programa de cómputo AXIL (por sus siglas en inglés Analisis of $\mathrm{X}$ ray spectra by Iterative Least squares fitting). La concentración de los elementos detectados se obtiene con un programa del sistema QXAS (por sus siglas en inglés Quantitative X-Ray Analysis System), conocido como DCCR (por sus siglas en inglés Direct Comparison of Count Rates). El DCCR utiliza una función de sensibilidades, que se basa en estándares de sedimento, para calcular la concentración de cada elemento. Por otro lado, las concentraciones de aquellas muestras que dieron menos de la Cantidad Mínima Detectable del equipo, fueron descartados para el análisis estadístico.

El análisis estadístico se realizó con los paquetes MINITAB 16 y MYSTAT 12. El análisis requiere que los datos tengan varianza constante y sean distribuidos normalmente (Schropp \& Windom 1988, Schropp et al. 1990, Soto-Jiménez \& Páez-Osuna 2000). La transformación de los datos al logaritmo de la razón de concentraciones elemento/aluminio cumple 

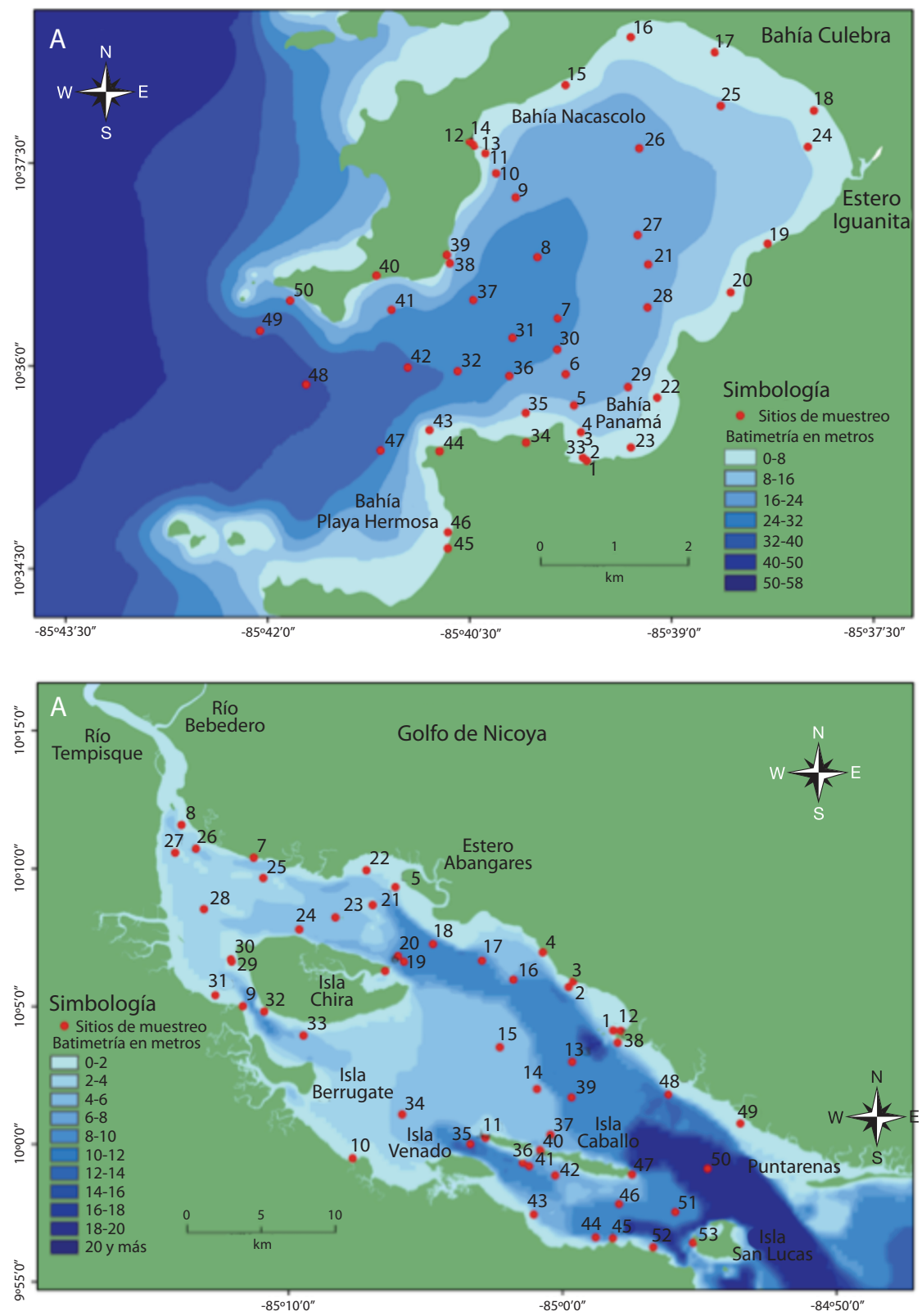

Fig. 1. Estaciones de muestreo en (A) Bahía Culebra y (B) Golfo de Nicoya.

Fig. 1. Sampling stations at (A) Bahía Culebra and (B) Golfo de Nicoya.

con esta condición (Schropp \& Windom 1988). Utilizando esta transformación y el criterio de normalidad de Anderson-Darling, se evaluaron los datos del logaritmo de la razón de concentraciones elemento/aluminio y se eliminaron los valores que excedieron los límites del 95\% de normalidad, hasta obtener al menos el 50\% de los datos originales cumpliendo con la prueba de normalidad. Si menos del $50 \%$ de los elementos originales no cumple con esta 
prueba, se considera que hay tres posibilidades: 1- una contaminación de muestrea al tomarla o manipularla, 2- un error en el análisis de laboratorio, y 3- enriquecimiento del elemento. Una vez rechazada o aprobada la hipótesis de normalidad, se realizó una regresión lineal del logaritmo de la concentración de cada elemento como variable dependiente y el logaritmo de la concentración del aluminio como variable independiente. Para conformar estadísticos robustos (Blank 1980) de la regresión lineal, en algunos casos otros elementos fueron eliminados, de manera que se cumpliera con la normalidad de los residuos, una distribución $\mathrm{t}_{(\alpha=0,05 \text {; }}$ ${ }_{25)}>2.06$, y una razón de varianza Fisher: $F>$ 4. El límite de predicción superior (LPS) y el límite de predicción inferior (LPI) al 95\% de la regresión lineal, fueron determinados para cada elemento. Estos límites representan el ámbito posible de los logaritmos de la concentración del metal en ambientes considerados "limpios" o no contaminados.

\section{RESULTADOS}

Bahía Culebra: Los principales valores de estadísticos de las concentraciones de los elementos estudiados en Bahía Culebra se muestran en el Cuadro 1. De este análisis sobresale la gran variabilidad en la concentración de los elementos cromo y el estroncio.

La Cantidad Mínima Detectable de los elementos de la Tabla 1 es el siguiente: aluminio $=0.01 \%$, potasio $=0.15 \%$, calcio $=0.08 \%$, manganeso $=0.004 \%$, Titanio $=0.02 \%$, Hierro $=0.004 \mathrm{mg} / \mathrm{Kg}$, cromo $=89 \mathrm{mg} / \mathrm{kg}$, cobre $=32$ $\mathrm{mg} / \mathrm{kg}$, zinc $=12 \mathrm{mg} / \mathrm{kg}$, rubidio $=4 \mathrm{mg} / \mathrm{kg}$, estroncio $=3 \mathrm{mg} / \mathrm{kg}$ y plomo $=2 \mathrm{mg} / \mathrm{kg}$.

El análisis de normalización de la razón del logaritmo de las concentraciones elemento/ aluminio se resume en el Cuadro 2. El número de muestras que cumplieron con un valor $\mathrm{p}>$ 0.05 se muestran bajo la columna $\mathrm{N}$ (Cuadro 2). El estroncio y el manganeso fueron los elementos que se le tuvieron que eliminar el número mayor de muestras (29 y 27 respectivamente) para cumplir con la hipótesis de normalidad, quedando menos del $50 \%$ de las muestras originalmente analizadas, por lo que estos elementos serán descartados para construir alguna relación de enriquecimiento/ contaminación con el aluminio. Las distribuciones del logaritmo del titanio/aluminio y el

CUADRO 1

Valores de variables estadísticas de las concentraciones de los metales en sedimentos marinos de Bahía Culebra

TABLE 1

Values of statistical variables of the concentrations of metals in marine sediments from Bahía Culebra

\begin{tabular}{|c|c|c|c|c|c|}
\hline Elem & $\mathrm{N}$ & Promedio & DesvSt & Mínimo & Máximo \\
\hline $\operatorname{Al}(\%)$ & 50 & 5.23 & 2.32 & 0.45 & 8.93 \\
\hline $\mathrm{K}(\%)$ & 50 & 0.97 & 0.66 & 0.12 & 4.16 \\
\hline $\mathrm{Ca}(\%)$ & 50 & 15.87 & 12.45 & 1.02 & 55.79 \\
\hline $\operatorname{Mn}(\%)$ & 50 & 0.094 & 0.050 & 0.004 & 0.236 \\
\hline $\operatorname{Ti}(\%)$ & 50 & 0.54 & 0.31 & 0.02 & 1.61 \\
\hline $\mathrm{Fe}(\%)$ & 50 & 5.400 & 2.619 & 0.194 & 13.410 \\
\hline $\mathrm{Cr}(\mathrm{mg} / \mathrm{Kg})$ & 43 & 302 & 196 & 89 & 805 \\
\hline $\mathrm{Cu}(\mathrm{mg} / \mathrm{kg})$ & 50 & 33 & 15 & 16 & 84 \\
\hline $\mathrm{Zn}(\mathrm{mg} / \mathrm{kg})$ & 50 & 71 & 22 & 26 & 142 \\
\hline $\mathrm{Rb}(\mathrm{ppm})$ & 49 & 12 & 7 & 4 & 35 \\
\hline $\mathrm{Sr}(\mathrm{mg} / \mathrm{kg})$ & 50 & 782 & 562 & 141 & 2513 \\
\hline $\mathrm{Pb}(\mathrm{mg} / \mathrm{kg})$ & 50 & 9 & 5 & 3.0 & 33 \\
\hline
\end{tabular}

$\mathrm{N}$ : número de muestras, DesvSt: desviación estándar, \%: porcentaje.

$\mathrm{N}$ : number of samples, DesvSt: standard deviation, \%: percentage, minimum and maximum. 
CUADRO 2

Normalización del logaritmo de la concentración del elemento con respecto a la de aluminio en Bahía Culebra

TABLE 2

Normalized logarithm of the element concentration with respect to aluminum at Bahía Culebra

\begin{tabular}{ccccc} 
Elem/Al & DesvSt & $\mathrm{N}$ & $\mathrm{AD}$ & $\mathrm{p}^{* *}$ \\
$\mathrm{~K} / \mathrm{Al}$ & 0.33 & 46 & 0.42 & 0.32 \\
$\mathrm{Ca} / \mathrm{Al}$ & 0.46 & 26 & 0.69 & 0.06 \\
$\mathrm{Mn} / \mathrm{Al}$ & 0.09 & 23 & 0.72 & 0.05 \\
$\mathrm{Ti} / \mathrm{Al}$ & 0.56 & 29 & 0.68 & 0.07 \\
$\mathrm{Fe} / \mathrm{Al}$ & 3.66 & 46 & 0.58 & 0.13 \\
$\mathrm{Cr} / \mathrm{Al}$ & 0.73 & 29 & 0.67 & 0.07 \\
$\mathrm{Cu} / \mathrm{Al}$ & 0.38 & 26 & 0.67 & 0.07 \\
$\mathrm{Zn} / \mathrm{Al}$ & 0.26 & 37 & 0.56 & 0.14 \\
$\mathrm{Rb} / \mathrm{Al}$ & 0.33 & 39 & 0.48 & 0.22 \\
$\mathrm{Sr} / \mathrm{Al}$ & 1.26 & 21 & 0.63 & 0.09 \\
$\mathrm{~Pb} / \mathrm{Al}$ & 0.13 & 31 & 0.38 & 0.39 \\
\hline
\end{tabular}

* Estadístico de Anderson-Darling (AD)

** valor $\mathrm{p}<0.05$ : rechaza la hipótesis de normalidad.

estroncio/aluminio no mostraron estadísticos robustos en la regresión lineal como se verá más adelante.

El potasio y el hierro fueron los elementos cuyas concentraciones, en mayor número, se ajustaron a la hipótesis de normalidad, siendo la distribución de potasio más normal de estos, según lo indica el estadístico AD. De todos los elementos estudiados, el plomo fue el elemento que mejor ajustó a esta distribución normal, como se puede ver a través del valor más bajo del estadístico AD (Cuadro 2). La distribución menos normal de todos ellos es del elemento manganeso, seguido por el calcio, el titanio, el cobre y el cromo.

Una vez rechazada o aceptada la hipótesis de normalidad, se realizó la regresión lineal de cada elemento, utilizando como variable dependiente el logaritmo de la concentración del elemento y el logaritmo de la concentración del aluminio como variable independiente. Fueron eliminados los valores que fueron identificados atípicos (“outlier" en inglés) por el programa MYSTAT en la regresión lineal. En algunos casos, aquellas concentraciones de los elementos ligeramente fuera del límite de predicción superior (LSP) e inferior (LPI) de la regresión lineal, se dejaron para mantener el mayor valor de la t de Student y hacer más significativa su relación estadística.

La distribución de los logaritmos de las concentraciones de los metales titanio y el estroncio, no muestra una correlación significativa con la concentración del aluminio, lo cual se puede ver a través de los estadísticos t y F (Cuadro 3). Los logaritmos de las concentraciones de potasio, calcio y el manganeso, produjeron correlaciones negativas con la concentración del aluminio. Los logaritmos de las concentraciones de los elementos rubidio, plomo, zinc y el hierro (Cuadro 3) tienen correlaciones positivas y muy significativas con la concentración del aluminio.

Las ecuaciones que se derivan de los parámetros del Cuadro 3, y que gráficamente se representan en las Figs. 2 y 3, constituyen la herramienta propuesta para establecer la relación enriquecimiento/contaminación de estos elementos (Figs. 2, 3) en Bahía Culebra.

No hubo correlación entre el logaritmo de las concentraciones del titanio con la concentración del aluminio, lo cual se nota por el valor de estadísticos $\mathrm{t}<2$ y $\mathrm{F}<4$ (Cuadro 2), por lo que este elemento tampoco pueden ser 
CUADRO 3

Regresión del logaritmo de las concentraciones de los elementos respecto al aluminio en Bahía Culebra

TABLE 3

Regression of the logarithm of element concentration with respect to aluminum at Bahía Culebra

\begin{tabular}{|c|c|c|c|c|c|}
\hline Elem & $\mathrm{N}$ & b & $\mathrm{m}$ & $\mathrm{t}$ & F \\
\hline $\mathrm{K}(\%)$ & 44 & 0.12 & -0.27 & -2.04 & 4.18 \\
\hline $\mathrm{Ca}(\%)$ & 41 & 1.72 & -0.89 & -3.99 & 15.96 \\
\hline $\operatorname{Ti}(\%)$ & 28 & -0.37 & 0.10 & 0.06 & 0.38 \\
\hline $\mathrm{Fe}(\%)$ & 45 & 2.08 & 4.13 & 4.22 & 20.49 \\
\hline $\mathrm{Cr}(\mathrm{mg} / \mathrm{Kg})$ & 29 & 1.86 & 0.84 & 2.25 & 5.07 \\
\hline $\mathrm{Cu}(\mathrm{mg} / \mathrm{Kg})$ & 26 & 1.08 & 0.60 & 3.02 & 9.11 \\
\hline $\mathrm{Zn}(\mathrm{mg} / \mathrm{Kg})$ & 35 & 1.38 & 0.59 & 5.08 & 25.81 \\
\hline $\mathrm{Rb}(\mathrm{mg} / \mathrm{Kg})$ & 39 & 0.50 & 0.80 & 5.0 & 25.00 \\
\hline $\mathrm{Pb}(\mathrm{mg} / \mathrm{Kg})$ & 31 & 0.30 & 0.81 & 5.81 & 34.23 \\
\hline
\end{tabular}

$\mathrm{N}$ : número de muestras, b: intercepto, m: pendiente, t: prueba de Student y F: prueba de Fisher.

$\mathrm{N}$ : number of samples, b: intercept, m: slope, t: Student test and F: Fisher test.
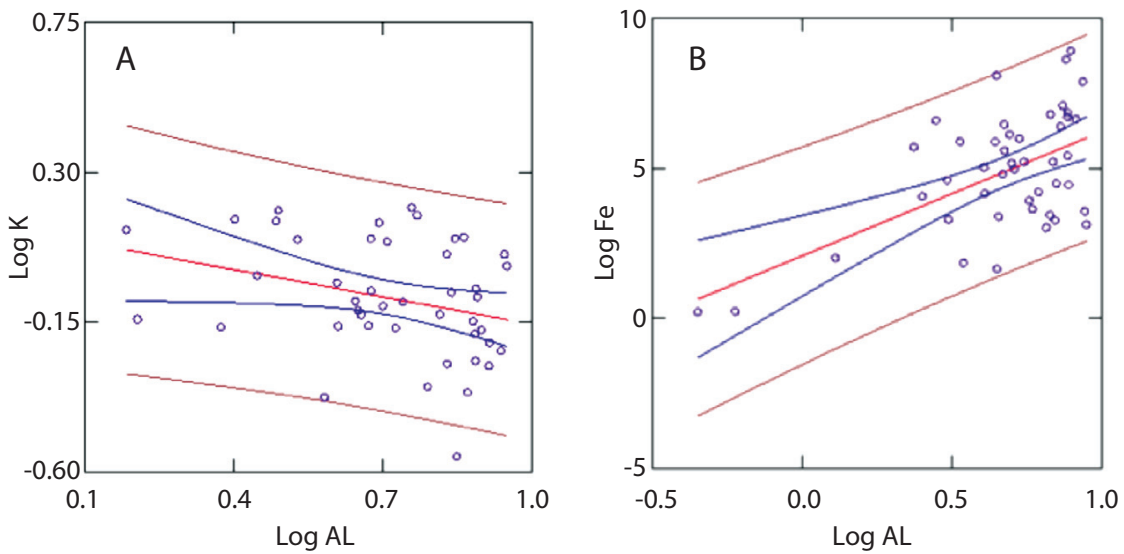

Fig. 2. Regresión lineal y los límites de predicción superior (LPS) e inferior (LPI) (líneas violeta), y los intervalos de confianza (líneas azules) de los elementos en \% respecto al Aluminio (Al) de los elementos en ppm en Bahía Culebra. (A) Potasio y (B) Hierro.

Fig. 2. Linear regression and predicted upper and lower limits (purple lines), and the confidence limits (blue lines) of the elements in \% with respect to Aluminum (Al) in Bahía Culebra. (A) Potassium and (B) Iron.

utilizados para determinar contaminación en este estudio.

Golfo de Nicoya: El análisis estadístico y de normalización de las concentraciones de los elementos correspondiente a los sedimentos marinos del Golfo de Nicoya se muestra en los Cuadros 4 y 5 .
La distribución del cobre no se ajustó a una distribución normal, como lo muestra el estadístico $\mathrm{p}<0.05$ en el Cuadro 5. Además, menos del $50 \%$ de las muestras se mantienen en la distribución, por lo que no podrá ser utilizado para elaborar alguna relación con el aluminio. El análisis también demuestra que para el elemento calcio, se tuvieron que eliminar 

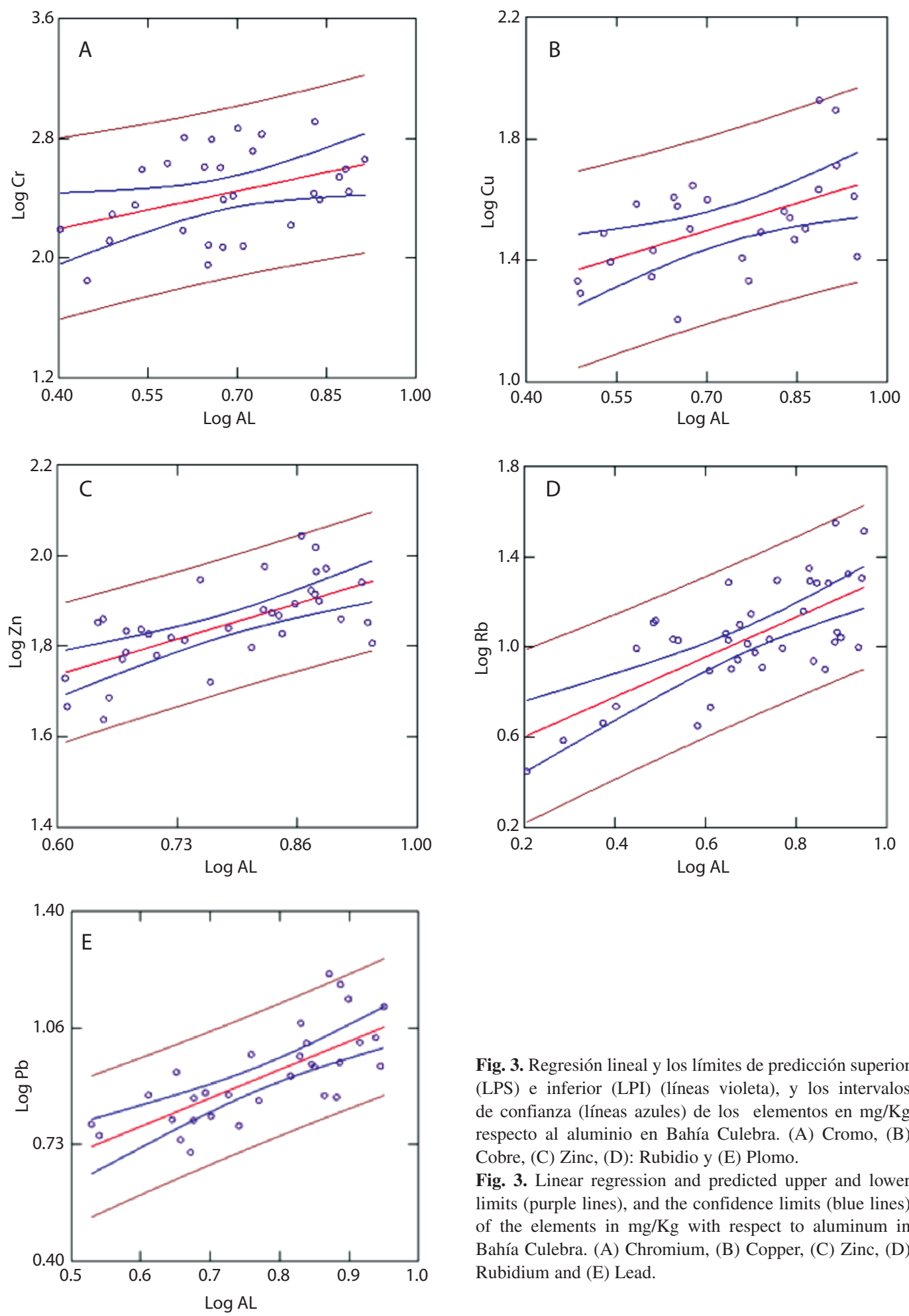

Fig. 3. Regresión lineal y los límites de predicción superior (LPS) e inferior (LPI) (líneas violeta), y los intervalos de confianza (líneas azules) de los elementos en $\mathrm{mg} / \mathrm{Kg}$ respecto al aluminio en Bahía Culebra. (A) Cromo, (B) Cobre, (C) Zinc, (D): Rubidio y (E) Plomo.

Fig. 3. Linear regression and predicted upper and lower limits (purple lines), and the confidence limits (blue lines) of the elements in $\mathrm{mg} / \mathrm{Kg}$ with respect to aluminum in Bahía Culebra. (A) Chromium, (B) Copper, (C) Zinc, (D) Rubidium and (E) Lead. 
CUADRO 4

Valores de variables estadísticas de las concentraciones de los metales en sedimentos marinos deL Golfo de Nicoya

TABLE 4

Values of statistical variables of the concentrations of metals in marine sediments from the Golfo de Nicoya

\begin{tabular}{|c|c|c|c|c|c|}
\hline Elem & $\mathrm{N}$ & Promedio & DesvSt & Mínimo & Máximo \\
\hline $\mathrm{Al}(\%)$ & 53 & 6.91 & 2.23 & 0.97 & 10.45 \\
\hline $\mathrm{K}(\%)$ & 47 & 0.90 & 0.69 & 0.15 & 4.23 \\
\hline $\mathrm{Ca}(\%)$ & 53 & 5.84 & 10.60 & 0.64 & 49.19 \\
\hline $\operatorname{Ti}(\%)$ & 53 & 0.35 & 0.16 & 0.01 & 0.83 \\
\hline $\operatorname{Mn}(\%)$ & 53 & 0.099 & 0.046 & 0.020 & 0.270 \\
\hline $\mathrm{Fe}(\%)$ & 53 & 4.41 & 1.69 & 0.37 & 8.94 \\
\hline $\mathrm{Cr}(\mathrm{mg} / \mathrm{Kg})$ & 32 & 253 & 297 & 83 & 1245 \\
\hline $\mathrm{Cu}(\mathrm{mg} / \mathrm{Kg})$ & 53 & 51 & 18 & 17 & 87 \\
\hline $\mathrm{Zn}(\mathrm{mg} / \mathrm{Kg})$ & 53 & 67 & 23 & 16 & 162 \\
\hline $\mathrm{Rb}(\mathrm{mg} / \mathrm{Kg})$ & 53 & 27 & 9 & 9 & 59 \\
\hline $\mathrm{Sr}(\mathrm{mg} / \mathrm{Kg})$ & 53 & 407 & 458 & 151 & 2314 \\
\hline $\mathrm{Pb}(\mathrm{mg} / \mathrm{Kg})$ & 53 & 12 & 6 & 2 & 44 \\
\hline
\end{tabular}

N: número de muestras, DesvSt: desviación estándar, \%: porcentaje,

N: number of samples, Prom: average, DesvSt: standard deviation, \%: percentage, minimum and maximum.

\section{CUADRO 5}

Normalización del logaritmo de la concentración del elemento respecto del aluminio en el Golfo de Nicoya

TABLE 5

Normalized logarithm of the element concentration respect to aluminum at the Golfo de Nicoya

\begin{tabular}{ccccc} 
Elem/Al & DesvSt & $\mathrm{N}$ & $\mathrm{AD}$ & $\mathrm{p}$ \\
$\mathrm{K} / \mathrm{Al}$ & 0.09 & 37 & 0.49 & 0.21 \\
$\mathrm{Ca} / \mathrm{Al}$ & 0.26 & 26 & 0.69 & 0.07 \\
$\mathrm{Mn} / \mathrm{Al}$ & 0.15 & 45 & 0.37 & 0.50 \\
$\mathrm{Ti} / \mathrm{Al}$ & 0.13 & 43 & 0.68 & 0.07 \\
$\mathrm{Fe} / \mathrm{Al}$ & 0.12 & 46 & 0.58 & 0.12 \\
$\mathrm{Cr} / \mathrm{Al}$ & 0.38 & 27 & 1,06 & 0,01 \\
$\mathrm{Cu} / \mathrm{Al}$ & 0.32 & 18 & 1.31 & $<0.05$ \\
$\mathrm{Zn} / \mathrm{Al}$ & 0.13 & 40 & 0.29 & 0.60 \\
$\mathrm{Rb} / \mathrm{Al}$ & 0.12 & 41 & 0.39 & 0.37 \\
$\mathrm{Sr} / \mathrm{Al}$ & 0.23 & 42 & 0.26 & 0.67 \\
$\mathrm{~Pb} / \mathrm{Al}$ & 0.13 & 47 & 0.36 & 0.42 \\
\hline
\end{tabular}

más del $50 \%$ de las muestras para que cumpla con la prueba de normalización, por lo que tampoco podrá ser usado para establecer una ecuación con el aluminio. La distribución del Sr es la que cumplió mejor con una distribución normal (menor valor de AD, Cuadro 5). Sin embargo, tampoco tuvo una correlación con el aluminio ( $\mathrm{T}<2$, Cuadro 6). Con alto porcentaje de normalidad le siguen el zinc y el plomo. (Cuadro 5). De estos últimos, solo el plomo tiene una correlación positiva con el aluminio (Cuadro 6). El que más muestras mantiene en la distribución normal es el plomo, seguido por el hierro y el manganeso (Cuadro 5).

Un resumen del análisis de regresión lineal de estos elementos respecto del aluminio se muestra en el Cuadro 6.

El logaritmo de la concentración de los elementos potasio, manganeso, titanio y estroncio, mostraron una correlación negativa con la concentración del aluminio (Cuadro 6). La distribución del plomo tiene la correlación positiva más significativa, seguida por el cobre, como se deduce de los estadísticos t y $\mathrm{F}$ del Cuadro 6. Aunque la distribución del cobre debe descartarse como patrón indicador de contaminación, porque como se mencionó anteriormente, no cumplió con la prueba de normalización. En valor del estadístico t y $\mathrm{F}$, le sigue la distribución de hierro con una correlación significativa con aluminio (Cuadro 6). 
CUADRO 6

Regresión del logaritmo de la concentración de los elementos respecto al aluminio en el Golfo de Nicoya

TABLE 6

Regression of logarithm of elements concentration with respect to aluminum at the Golfo de Nicoya

\begin{tabular}{cccccc} 
Elemento & $\mathrm{N}$ & $\mathrm{b}$ & $\mathrm{m}$ & $\mathrm{t}$ & $\mathrm{F}$ \\
$\mathrm{K}(\%)$ & 37 & 0.10 & -0.28 & -1.93 & -4.15 \\
$\mathrm{Mn}(\%)$ & 23 & -0.63 & -0.12 & -2.14 & 17.21 \\
$\mathrm{Ti}(\%)$ & 43 & 0.11 & -0.58 & 3.62 & 4.57 \\
$\mathrm{Fe}(\%)$ & 46 & 0.20 & 0.52 & 2.13 & 13.09 \\
$\mathrm{Cr}(\mathrm{ppm})$ & 39 & 1.20 & 0.09 & 0.30 & 4.54 \\
$\mathrm{Cr}(\mathrm{mg} / \mathrm{Kg})$ & 19 & 2.30 & 0.20 & 0.36 & 0.10 \\
$\mathrm{Zn}(\mathrm{mg} / \mathrm{Kg})$ & 38 & 1.77 & 0.08 & 0.19 & 0.13 \\
$\mathrm{Rb}(\mathrm{mg} / \mathrm{Kg})$ & 41 & 1.41 & 0.05 & -1.03 & 0.04 \\
$\mathrm{Sr}(\mathrm{mg} / \mathrm{Kg})$ & 41 & 2.72 & -0.39 & 8.87 & 1.06 \\
$\mathrm{~Pb}(\mathrm{mg} / \mathrm{Kg})$ & 47 & 0.26 & 0.91 & & 78.72 \\
\hline
\end{tabular}

N: número de muestras, intercepto "b", pendiente "m", "t" de Student, "F" de Fisher.

$\mathrm{N}$ : number of samples, b: intercept, m: slope, t Student, and Fisher's F.

Las relaciones del logaritmo de las concentraciones de los elementos con el aluminio se observan gráficamente en las Figuras 4 y 5. Estas figuras también pueden ser utilizadas para determinar la relación de enriquecimiento/ contaminación en el Golfo de Nicoya.

Las relaciones del logaritmo de las concentraciones del metal-aluminio en sedimentos naturales como los de Bahía Culebra y el Golfo de Nicoya son descritas por las ecuaciones lineales sencillas de regresión lineal (Cuadros 3 y 6), que relacionan la concentración del elemento con respecto de la concentración del aluminio de la siguiente manera:

$\log [$ elemento $]=b+\mathrm{m} * \log [$ aluminio $]$

Donde,

Log[elemento]: logaritmo de la concentración del elemento

b: intercepto

m: pendiente

Log[aluminio]: logaritmo de la concentración del aluminio

Por ejemplo: en la estación 1 del Golfo de Nicoya, la concentración de aluminio fue de
$0.97 \%$. Aplicando la ecuación anterior según los parámetros del Cuadro 6:

$$
\log [\text { plomo }]=0.262+0.911 * \log [0.97]=0.25 \mathrm{mg} / \mathrm{Kg}
$$

Si se grafica en la Fig. 5-F el Log[plomo]= $0.25 \mathrm{mg} / \mathrm{Kg}$ y el $\log$ [aluminio] $=0.97 \%$, el punto estaría fuera (debajo en este caso) del límite de predicción inferior (LPI) del 95\%. Esto indicaría una relación no natural del plomo en el Golfo de Nicoya. De esta manera, la regresión lineal y los límites de predicción superior (LSP) e inferior (LPI) pueden ser utilizados para determinar rutinariamente si muestras de sedimentos de estos estuarios son enriquecidas con metales o no cumplen con una relación natural con $\mathrm{Al}$.

\section{DISCUSIÓN}

Bahía Culebra: El Cuadro 7 resume valores de concentraciones promedio de los elementos en las rocas y en las arcillas de los sedimentos marinos, como también referencias de estudios anteriores y de lugares considerados contaminados (Carvalho et al. 2002, 

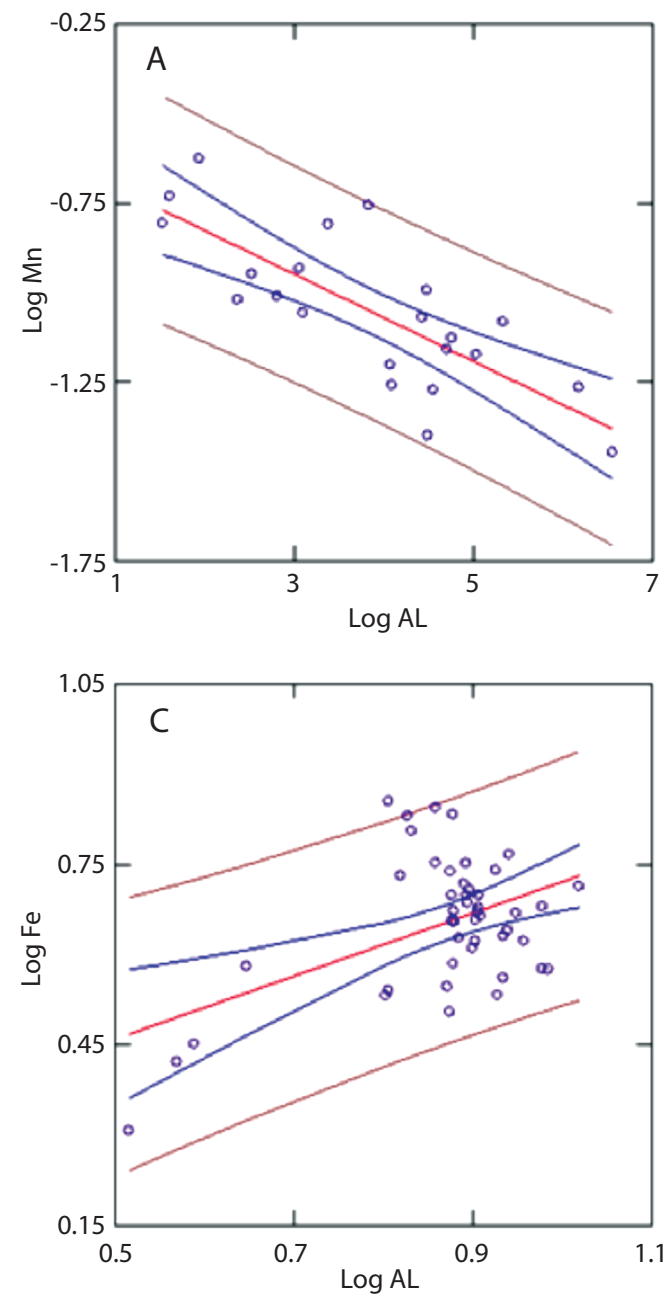

Pereira et al. 2007, Graham \& Semmes 2005, Kress et al. 1998, García-Céspedes et al. 2004), con los cuales se pueden evaluar las concentraciones medidas en este estudio. Utilizando la información del Cuadro 1 y del Cuadro 7, se nota que las concentraciones de aluminio y manganeso encontradas en Bahía Culebra están en el ámbito en que se encuentran en las rocas o en las arcillas de sedimentos marinos. El hierro tiene un valor promedio ligeramente mayor que el de referencia en las rocas y arcillas, y supera los ámbitos en algunas muestras (Cuadro 1), de los encontrados en este mismo lugar por García-Céspedes et al. (2004) (Cuadro 7). El

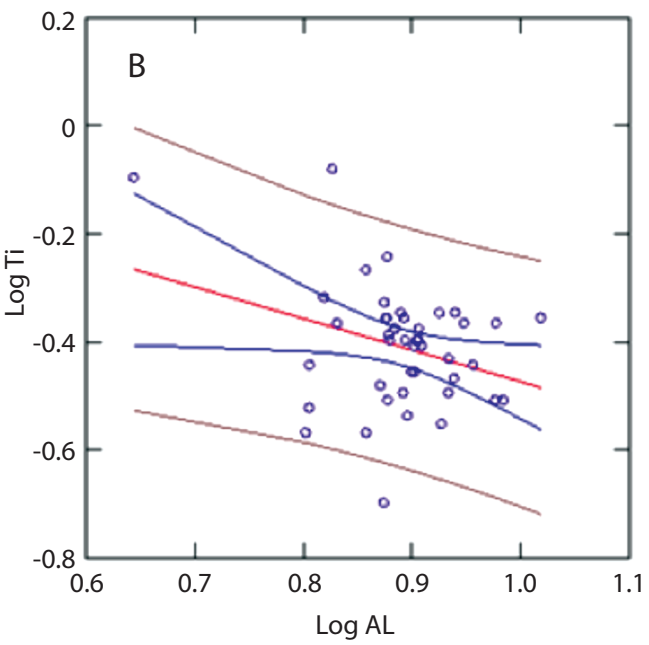

Fig. 4. Regresión lineal y los límites de predicción superior (LPS) e inferior (LPI) (líneas violeta), y los intervalos de confianza (líneas azules) de los elementos en \% respecto al aluminio en el Golfo de Nicoya. (A) Manganeso y (B) Titanio y (C) Hierro.

Fig. 4. Linear regression and predicted upper and lower limits (purple lines), and the confidence limits (blue lines) of the elements in \% with respect to aluminum in Golfo de Nicoya. (A) Manganese, (B) Titanium and (C) Iron.

cromo muestra valores promedio más altos que los valores de referencia en las rocas y en las arcillas, e incluso supera los valores reportados por Carvalho et al. (2002) (Cuadro 7) de una bahía contaminada. Otros elementos enriquecidos son el calcio, manganeso y el estroncio, que no superaron más del $50 \%$ de las muestras en el logaritmo de la concentración de la razón con el aluminio. El logaritmo de la concentración del titanio tampoco cumple con los estadísticos de la regresión lineal (Cuadro 3). Por esto, estos elementos no deben ser usados para encontrar una relación de enriquecimiento/contaminación con el aluminio. 

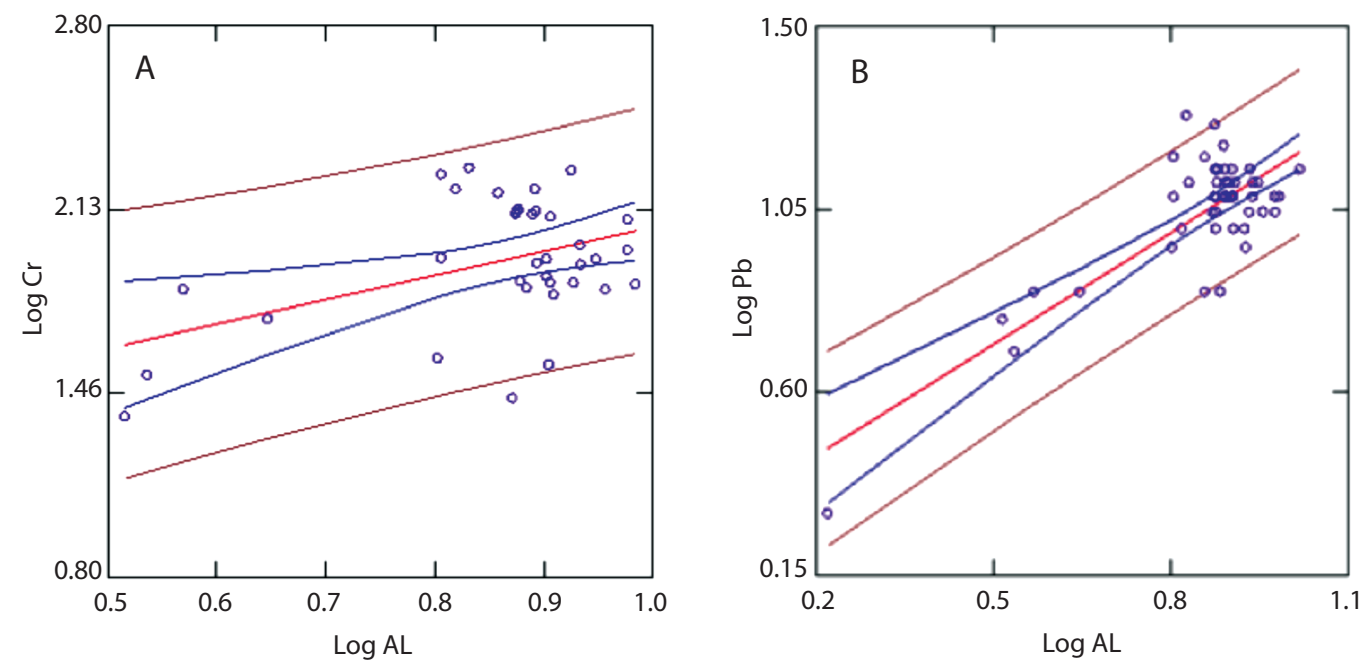

Fig. 5. Regresión lineal y los límites de predicción superior (LPS) e inferior (LPI) (líneas violeta), y los intervalos de confianza (líneas azules) de los elementos en $\mathrm{mg} / \mathrm{Kg}$ respecto al aluminio en el Golfo de Nicoya. (A) Cromo y (B) Plomo. Fig. 5. Linear regression and predicted upper and lower limits (purple lines), and the confidence limits (blue lines) of the elements in $\mathrm{mg} / \mathrm{Kg}$ with respect to aluminum in Golfo de Nicoya. (A) Chromium and (B) Lead.

CUADRO 7

Concentraciones de los elementos como referencia

TABLE 7

Concentrations of elements as reference

\begin{tabular}{|c|c|c|c|c|c|c|}
\hline Elem & Rocas $^{1}$ & Arcillas $^{2,3}$ & B. contam ${ }^{2}$ & G. Nicoya ${ }^{4}$ & G. Nicoya ${ }^{5}$ & B. Culebra ${ }^{5}$ \\
\hline $\mathrm{AL}(\%)$ & 6.9 & 8.1 & $* *$ & & & \\
\hline $\mathrm{K}(\%)$ & $* *$ & $* *$ & $* *$ & & & \\
\hline $\mathrm{Ca}(\%)$ & $* *$ & $* *$ & $* *$ & & & \\
\hline $\operatorname{Mn}(\%)$ & 0.070 & 0.085 & $* *$ & $0.089-0.082$ & & \\
\hline $\operatorname{Ti}(\%)$ & $* *$ & $* *$ & $* *$ & & & \\
\hline $\mathrm{Fe}(\%)$ & 3.6 & 4.7 & $* *$ & $4.61-4.79$ & $2.67-5.07$ & $2.48-7.33$ \\
\hline $\mathrm{Cr}(\mathrm{mg} / \mathrm{Kg})$ & 71 & 90 & 169.3 & & & \\
\hline $\mathrm{Cu}(\mathrm{mg} / \mathrm{Kg})$ & 32 & 5 & 225.1 & $58.5-68.1$ & $44.5-81.7$ & $32.3-84.7$ \\
\hline $\mathrm{Zn}(\mathrm{mg} / \mathrm{Kg})$ & 127 & 95 & 657.8 & $83.1-84.1$ & $56.9-89.3$ & $50.3-96.9$ \\
\hline $\mathrm{Rb}(\mathrm{mg} / \mathrm{Kg})$ & $* *$ & $* *$ & $* *$ & & & \\
\hline $\mathrm{Sr}(\mathrm{mg} / \mathrm{Kg})$ & $* *$ & $* *$ & $* *$ & & & \\
\hline $\mathrm{Pb}(\mathrm{mg} / \mathrm{Kg})$ & 16 & 20.0 & 180.0 & $15.2-18.3$ & $1.6-8.2$ & $1.0-3.8$ \\
\hline
\end{tabular}

B.contam: Bahía contaminada.

1. Carvalho et al. (2002)

2. Pereira et al. (2007)

3. Graham \& Semmes (2005)

4. Kress et al. (1998)

5. García-Céspedes et al. (2004) 
Golfo de Nicoya: Las concentraciones de aluminio, manganeso, cobre, rubidio y plomo tienen promedios mayores en esta región que en Bahía Culebra. Sin embargo, sus concentraciones están alrededor de los valores de referencia de las rocas, en los sedimentos marinos, y en el ámbito de valores de otras mediciones en la región (Cuadro 7). El cromo presenta promedios muy altos, con concentraciones que superan los valores de bahías contaminadas (Pereira et al. 2007). Fuller et al. (1989) identificó altas concentraciones de dicho elemento en el río Grande de Tárcoles producto de las descargas de curtidoras de cuero. El calcio y el cobre no superaron la normalización con más del 50\% de número de muestras (Cuadro 5), por lo que no se pudo encontrar una relación estadística de estos elementos con el aluminio.. La distribución del logaritmo de las concentraciones del zinc, rubidio y estroncio, tienen poca correlación con el aluminio, la cual se identifica desde los estadísticos (Cuadro 4), indicando esto, que hay muestras de estos elementos con concentraciones no naturales en el golfo.

Las concentraciones de los elementos en los sedimentos marinos son valorados si son naturales o enriquecidos de metal dependiendo de donde se ubica el logaritmo de la concentración del elemento y el logaritmo de la concentración del aluminio respecto de la regresión lineal y sus límites de predicción superior. Para esto se utilizan las figuras 2, 3, 4 y 5 . Si un punto está dentro de los límites de predicción, entonces la concentración del metal en el sedimento está dentro del ámbito natural esperado. Aquellas muestras en que las que el logaritmo de la concentración del metal exceden los límites superiores $95 \%$ de predicción, este es considerado enriquecido. Un hallazgo de enriquecimiento no es siempre indicativo de enriquecimiento antropogénico. Otras razones posibles para un metal que excede el $95 \%$ de límite de predicción incluye: 1) errores de procedimiento (por ejemplo, contaminación de muestra durante la recolección, manejo y durante el almacenamiento, y 2) la probabilidad que unas pocas muestras de áreas naturales contengan metales cuyas concentraciones exceden los 95\% de límites de predicción. Los puntos que están fuera de los límites de predicción deben ser interpretados a la luz de información adicional disponible sobre fuentes posibles de contaminación metálica, la información de otras estaciones cerca, y la evaluación del manejo de la muestra o errores analíticos como lo señala Schropp et al. (1990). En Bahía Culebra se tiene que las estaciones 1,2 y 3 están prácticamente en el mismo sitio (Fig. 1). Sin embargo, la concentración de plomo en la estación 1 fue de $33 \mathrm{mg} / \mathrm{kg}$ y en la 2 de $17 \mathrm{mg} / \mathrm{kg}$. El valor de $33 \mathrm{mg} / \mathrm{kg}$ debe ser eliminado para que la distribución sea normal. Por otro lado, la concentración de la estación 2, pasa la prueba de normalidad y se mantiene en la regresión lineal cumpliendo con sus respectivos valores estadísticos. Estas diferencias indican un error de manipulación de la muestra y/o error en el análisis de laboratorio. Otro ejemplo lo son las muestras del Golfo de Nicoya de las estaciones 2 y 3 (Fig. 1-B) que están prácticamente en el mismo sitio y cuyas concentraciones respectivas, por ejemplo de plomo y de aluminio, son respectivamente: $19-44 \mathrm{mg} / \mathrm{Kg}$ y $6.72-1.81 \%$. Los valores de plomo y aluminio de la estación 2, no cumplen con la condición normal del análisis estadístico, lo que demuestra que los valores de las concentraciones son atípicos en este caso. Estas diferencias de nuevo, evidencian un problema en el manejo de las muestras, o en el análisis.

\section{AGRADECIMIENTOS}

Al CONICIT que a través de los fondos FUNDES II financió parte de esta investigación. A la Vicerrectoría de Investigación de la Universidad de Costa Rica que financió también parte de esta investigación a través de los proyectos V.I. 808-B0-092, 808-B0-654, 808-A9-180, 805-A7-002 y al IAI-CRN2-050. A Eleazar Ruiz por su invaluable colaboración logística y de apoyo durante el desarrollo del muestreo. 


\section{RESUMEN}

Se propone un método para evaluar enriquecimiento de metales en sedimentos de Bahía Culebra y en el Golfo de Nicoya de Costa Rica a través de la normalización de los elementos y el aluminio usando la regresión lineal de las concentraciones de los elementos y el Aluminio. Las distribuciones de los elementos manganeso y el estroncio de Bahía Culebra no cumplen con las pruebas de normalización y de regresión lineal, indicando una distribución no natural o enriquecimiento de estos elementos en esta región. En el Golfo de Nicoya los elementos cobre, zinc, rubidio y el estroncio no cumplen ya sea con la hipótesis de normalidad o con la regresión lineal respecto al aluminio, indicando un posible enriquecimiento de estos elementos en esta región. La mayoría de las concentraciones de los elementos tanto en ambos sitios de muestreo, con excepción del cromo, se encuentran dentro de los ámbitos naturales encontrados en las rocas o en las arcillas de sedimentos marinos a nivel mundial, y dentro de los ámbitos de las concentraciones de otros estudios realizados en estas mismas regiones. El cromo muestra valores promedio más allá de las concentraciones naturales, siendo la concentración de algunas muestras en el Golfo de Nicoya hasta diez veces mayores que el valor de una bahía con alta contaminación de este elemento.

Palabras clave: concentración de metales, Bahía Culebra, Golfo de Nicoya, enriquecimiento, contaminación, Costa Rica, América Central.

\section{REFERENCIAS}

Ackerman, F., M. Bergmann \& G. Schleichertl. 1983. Monitoring heavy metals in coastal estuarine sediments - a question of grain size: $<20 \mathrm{~m}$ versus $<60 \mathrm{~m}$. Environ. Technol. Lett. 4: 317-328.

Blank, L. 1980. Statistical Procedures for Engineering, Management and Science. McGraw-Hill, Nueva York.

Carvalho, A., S.J. Schropp, G.M. Sloane, T.P. Biernacki, T.L. Seal. 2002. Development of an Interpretive Tool for Assessment of Metal Enrichment in Florida Freshwater Sediment. Florida Department of Environmental Protection, Tallahassee, Florida.

Cooke, T.D. \& D.D. Drury. 1998. Calabazas Creek Pilot Sediment Sampling Study. Proc. 1998 NWQMC Natl. Monit. Conf.; Monitoring Critical Foundations to Protect our Waters. Reno, Nevada.

Daskalakis, K.D. \& T.P. O’Connor. 1995. Normalization an elemental sediment contamination in the coastal United States. Environ. Sci. Technol. 29: 470-477.
Dean, H.K., D. Maurer, J.A. Vargas \& C.H. Tinsman. 1986. Trace metal concentrations in sediment and invertebrates from the Golfo de Nicoya, Costa Rica. Mar. Pollut. Bull. 17: 128-131.

Fonseca, A.C. 2006. Golfo de Papagayo. Capítulo XIV, P. 186-194. In V. Nielsen-Muñoz \& M.A. AlpizarQuesada (eds.). Ambientes Marino Costeros de Costa Rica. Conservation Internacional. San José, Costa Rica.

Fuller, C.C., J.A. Davis, D.J. Cain, P.J. Lamothe, T.L. Fries, G. Fernandez, J.A. Vargas \& M.M. Murillo. 1989. Distribution and transport of sediment-bound metal contaminants in the Rio Grande de Tárcoles, Costa Rica (Central America). Water Res. 24: 805-812.

García-Céspedes, J., J. Acuña-González \& J.A. VargasZamora. 2004. Metales traza en sedimentos costeros de Costa Rica. Rev. Biol. Trop. 52 (Supl. 3A): 51-60.

Graham, P.N. \& R.H. Semmes, 2005. Interpretation of anthropogenic enrichment of metal concentration in sediments from a Savannah Harbor Boat Slip. Proc. 2005 Georgia Water Res. Conf. University of Georgia, Athens, Georgia.

Goldberg, E.D., J.J. Griffin, V. Hodge, M. Koide \& H. Window. 1979. Pollution history of the Savannah River estuary. Environ. Sci. Technol. 15: 588-594.

Hanson, P., D. Evans, D. Colby \& V. Zdanowics. 1993. Assessment of elemental contamination in estuarine and coastal environments based on geochemical and statistical modeling of sediments. Mar. Environ. Res. 36: 237-266.

Horowitz, A.J. 1991. A Primer on Sediment-trace Element Chemistry. $2^{\text {nd }}$ Ed. Lewis Publishers, Chelsea, Michigan.

Kress, N., S. Leon \& C. Brenes. 1997. Ecological assessment of the Golfo de Nicoya, Costa Rica. IOLR Report H12/98. A.I.D. Grant Proyect Officer, San José, Costa Rica.

Lee, G.F. \& A. Jones-Lee. 1993. Sediment quality criteria: numeric chemical- vs. biological effects-based approaches, p. 389-400. Proc. Water Environ. Fed. Natl. Conf. Anaheim, California.

Loring, D.H. 1991. Normalization of heavy approach for the granulometric normalization of trace metal data. Mar. Chem. 26: 155-168.

Mata, A. \& O. Blanco. 1994. La Cuenca del Golfo de Nicoya, un reto al desarrollo sostenible. Universidad de Costa Rica, San José, Costa Rica. 
Pereira, E., J.A. Baptista-Neto, B.J. Smith \& J.J. McAllister. 2007. The contribution of heavy metal pollution derived from highway runoff to Guanabara Bay sediments - Rio de Janeiro / Brazil. An. Acad. Brasil. Ciên. 79: 739-750

Ryan, J.D. \& H. L. Windom. 1988. Ageochemical and statistical approach for assessing metal pollution in coastal sediments, P. 47-58. In U. Seeliger, L. D. de Lacerda \& S. R. Patchineelam (eds.). Metal in Coastal Environments of Latin America. Springer. Londres.

Shiff, K.C. \& S.B. Weisberg. 1999. Iron as a reference element for determining trace metal enrichment in Southern California coastal shelf sediments. Mar. Environ. Res. 48: 161-176.

Schropp, S.J. \& H.L. Windom. 1988. A Guide to the Interpretation of Metal Concentrations in Estuarine Sediments. Florida Department of Environmental Protection. Tallahassee, Florida.

Schropp, S.J., F.G. Lewis, H.L. Windom, J.D. Ryan, F.D Calder \& L.C. Burney. 1990. Interpretation of metal concentrations estuarine sediments of Florida using
Aluminum as a reference element. Estuaries 15: 227-235.

Soto-Jiménez, M.F. \& F. Páez-Osuna. 2001. Distribution and normalization of heavy metal concentrations in mangrove and lagoonal sediments from Mazatlán Harbor (SE Gulf of California). Estuar. Coast. Shelf Sci. 53: 259-274.

Trefry, J. H., S. Metz, R. Trocine, and T. A. Nelsen, 1985, A decline in lead

transported by the Mississippi River. Science, V. 230, No. 4724, pp. 439-441.

Weisberg, S.B., H.T. Wilson, D.G. Heimbuch, H.L. Windom \& J.K. Summers. 2000. Comparison of sediment metal: aluminum relationships between the Eastern and Gulf coasts of the United States. Environ. Monit. Assess. 61: 373-385.

Windom, H.L., S.J. Schropp, F.D. Caldner, J.D. Ryan, R.G. Smith Jr., L.C. Burney, F.G. Lewis \& C.H. Rawlinson. 1989. Natural trace metal concentrations in estuarine and coastal marine sediments of the Southeastern United States. Environ. Sci. Technol. 23: $314-320$. 
